\title{
Sorge mit Aussicht
}

\section{Das Zeitregime sozialer Begleitung zu Hause}

\author{
Wolf Rainer Wendt
}

Prof. Dr. Wolf Rainer Wendt ist

Vorsitzender der Deutschen Gesellschaft für Care und Case Management sowie Honorarprofessor der Eberhard Karls Universität Tübingen. Er ist Mitglied im Beirat der Blätter der Wohlfahrtspflege. E-Mailwendt@imails.de
Mit »Care " etabliert sich ein Verständnis Sozialer Arbeit, das die Lebenspraxis ihrer Klientel zum Bezugspunkt macht. Professioneller Beistand als verlässliche und ermöglichende Hilfe muss dabei von Fall zu Fall der persönlichen Lebensgestaltung der beteiligten Menschen angepasst werden.

Es sind persönliche Perspektiven, die uns im Leben bewegen. Vielen Menschen mangelt es an ihnen, und dieser Mangel drückt. Menschen sorgen sich um das, was auf sie in ihrem Leben zukommt. Sie beschäftigt, wie es auch in schwieriger Lage weitergehen kann. Was erwartet wird, bestimmt das Handeln. Man muss sich etwas vornehmen können.

Aussichtslosigkeit macht tatenarm: Das ist ein in der Sozialen Arbeit zu wenig beachteter Gesichtspunkt von Armut und Not. Nachgerade in der Begleitung von Menschen in ihrem häuslichen Leben von Eltern mit ihren Kindern, von Alleinlebenden, von chronisch kranken oder pflegebedürftigen Menschen - ist ein perspektivisches und auf einen zeitlichen Verlauf angelegtes Arbeiten erforderlich, um mit ihnen in ihrem eigenen Lebensprojekt voranzukommen.

\section{Erstreckungen häuslicher Lebensführung}

Sozialprofessionell wird in das Leben von Menschen interveniert. Aber viele Hilfen, die geleistet werden, beruhen allein auf der Feststellung, dass hier und jetzt ein Bedarf für sie besteht. Die zeitliche Erstreckung, in der dieser Bedarf auftritt, wird von Humandiensten im professionellen Handeln wenig berücksichtigt. Was vorher war und nachher sein wird, interessiert nur zur diagnostischen Herleitung dessen, was jetzt zu tun angebracht ist, und zur prognostischen Einschätzung, was sich damit erreichen lässt.

Demgegenüber spielt im Haushalt individueller Lebensführung die Zeit in ihrer
Erstreckung eine entscheidende Rolle: Die inneren und äußeren Verhältnisse der persönlichen und familiären Existenz sind zäh; man hat sich eingerichtet, hegt auch Erwartungen und disponiert in ihrer Perspektive für das weitere Dasein. Dessen persönlicher oder familiärer Entwurf sollte in der Anlage des dienstlichen Einsatzes aufgegriffen und berücksichtigt werden.

Wir haben einen grundsätzlichen Unterschied zwischen der institutionellen und professionellen Praxis und der Lebenspraxis ihrer Klientel zu bedenken. Die eine ist funktionaler Natur und auf eine bestimmte Aufgabenerfüllung und die dafür geeigneten Verrichtungen konzentriert. Die andere hingegen ist ein von individuellen Erfahrungen, Erlebnissen und Erwartungen emotional bestimmtes Alltagshandeln. Es bleibt in seinem subjektiven Situiertsein außerhalb jeder dienstlichen Praxis. Diese greift eine Problematik auf und befasst sich mit Versorgungsmängeln, Erziehungsschwierigkeiten, Depression, Überschuldung, dabei wohl wissend, dass der Grund für sie anderer Natur ist und in der Behandlung einzelner Probleme nicht erreicht wird. Die professionelle Praxis kann sich aber mit dieser Erkenntnis verstehend und sorgend in den Horizont begeben, in dem Menschen ihr Leben führen.

Solange Personen ihren eigenen Haushalt haben und in ihm bei andauernden Belastungen oder kritischen Entwicklungen Unterstützung in ihrer selbständigen Lebensführung brauchen, wird Ausdauer nicht nur von den Sorgenden selber, sondern auch in der mitsorgenden Begleitung verlangt. Sie folgt dem Einzelnen, wohin er geraten ist und wohin er aus seiner Lage heraus in seinem Ergehen kommen kann.

Anders hinter den Mauern einer Institution. $\mathrm{Zu}$ ihr gehören Dauerhaftigkeit und feste Abläufe. Begeben sich Menschen in eine stationäre Einrichtung, müssen sie sich dessen Zeitregime von der Aufnahme bis zur Entlassung fügen. Die 
Lebensperspektiven, welche die Insassen mitgebracht haben, interessieren die Institution in der Regel nicht. (In der Hilfeplanung vor einer Unterbringung wird man deshalb disponieren, wie sie zeitlich $\mathrm{zu}$ begrenzen ist und was danach kommen soll.)

\section{Im Möglichkeitshorizont handeln}

In zeitlicher Hinsicht hat eine feste Einrichtung eine präventive Funktion, indem sie Insassen von einem institutionell nicht vorgesehenen Verhalten (möglichst) abhält. Das kann nur während der Unterbringung funktionieren. Bei beratender, pädagogischer oder pflegerischer Begleitung von Menschen in ihrem eigenen Haushalt steht in präventiver Hinsicht nicht Vermeidung, sondern Ermöglichung im Vordergrund. Sie beginnt im persönlichen Lebensfeld bei der Handlungsfreiheit, die dem Einzelnen zu Hause und in den Bezügen seines Daseins eingeräumt ist: Was kann und will er - zu Hause, mit oder ohne Partner, im Familienkreis, beruflich, gesundheitlich, in Überwindung von Schwierigkeiten und zur Lösung von Problemen - machen und erreichen? Was trägt soziale Begleitung dazu bei?

Bei Multiproblemfamilien, mit denen Sozialarbeit häufig befasst ist, ergeben sich Möglichkeiten der Bewältigung und Lösung, wenn das ganze häusliche Geschehen erörtert wird. Bei diesen Familien besteht der Zusammenhang der Schwierigkeiten nicht nur in ihrer Verkettung hier und jetzt, sondern auch in ihrer gegenseitigen Fortzeugung.

In einer Familiengruppenkonferenz (Hansbauer u. a. 2009) wird das deutlich, und bei Lage der Dinge berücksichtigt die Entscheidungsfindung in der innerfamiliären Diskussion von vornherein abzusehende Entwicklungen. Eine formelle Hilfe zur Erziehung oder zur Versorgung lässt sich an diesen Erkundungsprozess im Familienrat anschließen. Dem familiären Treffen korrespondiert die Konsultation der Fachkräfte. Für den weiteren Verlauf kann sich eine Synchronisierung von Bemühungen in der Familie und von extern ergeben: Wer macht was wann mit wem und wozu? In der personenbezogenen Versorgung wird das Handeln von formeller Seite mit informellem Handeln in der Koordination auf Ziele hin verknüpft.

\section{Sorge als Lebensentwurf}

Der Mensch sorgt sich und trifft Dispositionen in dem, was er sich vornimmt. Sei es in Verfolgung alltagsübergreifender Strebungen oder bei Befürchtungen in der Ungewissheit, was kommen mag. Solange jemand sich alltäglich um eigenes und gemeinsames Ergehen kümmert, bleibt das Leben ein Entwurf. Aktuelle Probleme, die man zu bewältigen hat, werden in den Horizont der Lebensplanung gestellt und haben darin ihr Gewicht. Soll nun

\section{Wie die Menschen zur Sorge kamen - eine Fabel}

Als einst die Sorge über einen Fluss ging, sah sie tonhaltiges Erdreich, sinnend nahm sie davon ein Stück und begann es zu formen. Während sie bei sich darüber nachdenkt, was sie geschaffen, tritt Jupiter hinzu. Ihn bittet die Sorge, dass er dem geformten Stück Ton Geist verleihe. Das gewährt ihr Jupiter gern.

Als sie aber ihrem Gebilde nun ihren Namen beilegen wollte, verbot das Jupiter und verlangte, dass ihm sein Name gegeben werden müsse. Während über den Namen die Sorge und Jupiter miteinander stritten, erhob sich auch die Erde und begehrte, dass dem Gebilde ihr Name beigelegt werde, da sie ja doch ihm ein Stück ihres Leibes dargeboten habe.

Die Streitenden nahmen Saturn zum Richter. Und ihnen erteilte Saturn folgende anscheinend gerechte Entscheidung: »Du, Jupiter, weil du den Geist gegeben hast, sollst bei seinem Tod den Geist, du, Erde, weil du den Körper geschenkt hast, sollst den Körper empfangen. Weil aber die Sorge dieses Wesen zuerst gebildet, so möge, solange es lebt, die Sorge es besitzen. Weil aber über den Namen Streit besteht, so möge es >homo heißen, da es aus shumus< (Erde) gemacht ist."

220. Fabel von Hygin, ein lateinisches Werk aus dem 2. Jahrhundert nach Christus

aus dem System personenbezogener Dienste geholfen werden, ist die Eigensorge des Menschen nicht nur zu berücksichtigen, um Ressourcen zu sparen. Vielmehr geht es darum, worin und wohin eine Hilfe wirkt. Sie fließt bei einer momentanen Intervention in fortdauerndes Sorgen ein. Eine bedachte Hilfeplanung trifft in der Leistungsgestaltung auf individuelle Lebensplanung.

Sorge hat ihre Ausdehnung in der Zeit. Für den Philosophen Martin Heidegger ist die Sorge die existenziale Struktur des menschlichen Daseins. Wir finden in "Sein und Zeit « ausgeführt, dass der Mensch in der Sorge » sich selbst voraus « ist, vorweg schon in der Welt und in ihr entworfen auf Zukunft. Im Kontinuum der Sorge begegnet die Absicht einer Hilfe und Unterstützung den Menschen andauernd davon bewegt, wie sie sich selbst und ihren Verhältnissen ausgesetzt sind.

Folglich ist im Zeitregime formeller extramuraler Versorgung und sozialer Begleitung " zu Hause « auf die lebenszeitlichen Dispositionen von Klienten und von informell Beteiligten einzugehen. Das ist eine Anforderung an die Kommunikation zwischen ihnen und denen, die sich professionell engagieren. Gegenstand des Dialogs sind nicht nur Belange der unmittelbaren Hilfe, der Pflege oder der Erziehung, sondern man konsultiert einander in der individuellen und gemeinsamen Lebenslage und dem alltäglichen Gang der Dinge in ihr.

Die Problematik der Lebensführung lässt sich in einer Sorgeberatung (Wendt 2009) ungeteilt aufgreifen. Ist die professionelle Begleitung von jungen Menschen in einem Heim als "geteiltes Leben « bezeichnet worden, wird bei der Entfernung, die zwischen dem »Berufsleben « von Helfern und dem eigenen Leben von Klienten besteht, andauernd viel zur Überbrückung dieser Differenz zu tun sein.

\section{Verlässlich sein und Sicherheit bieten - auf Zeit}

Der Verlaufsform persönlicher Lebensgestaltung können unterstützende Hilfen und andere nötige Maßnahmen angepasst werden. Sie bieten so nicht zuletzt Verlässlichkeit und Sicherheit für diejenigen, die mit einer Unterstützung rechnen müssen und mit ihr etwas anfangen wollen. Beispielsweise ist, um Anforderungen 
in Beruf und Familie miteinander vereinbaren zu können, dies die Weise, in der den Eltern eine Kinderbetreuung angeboten werden soll. Auch nach Gewährung eines Persönlichen Budgets bedarf es flexibler Arrangements, die den individuellen Bedürfnissen von Budgetnehmern nachkommen.
Allgemein wird man in einer professionell gestalteten Versorgung - zum Beispiel mit Hilfen zur Erziehung oder bei häuslicher Pflege - die Diskrepanz von

\section{Was versteht man eigentlich unter Care Management?}

Wir sind vorübergehend nicht im Dienstzimmer anzutreffen.

\section{$\mathrm{Zu}$ den vereinbarten Zeiten stehen wir ihnen gerne wieder zur Verfügung. Danke für ihr Verständnis.}

Ihr Betreuungsteam

Care Management bezeichnet allgemein die Steuerung humandienstlicher Versorgung im Sozialwesen (social care) und im Gesundheitswesen (health care, medical care) mit dem Ziel einer Optimierung von Versorgungsprozessen. Der Begriff ist eingeführt worden, um im Bereich von Care - im Sinne der Fürsorge, Pflege, stationärer Behandlung und ambulanter Betreuung - die Aufgaben des Managements summarisch zu bezeichnen. Synonym wird auch der Begriff Versorgungsmanagement verwandt.

Im engeren Sinne betrifft das Care Management die betrieblichen Abläufe und das Versorgungshandeln in der organisierten Ausführung der Leistungserbringung auf der Ebene von Einrichtungen z. B. in einem Krankenhaus oder in einem Pflegeheim. Im weiteren Sinne ist das staatlich eingerichtete bzw. öffentlich zu verantwortende Management der humandienstlichen Prozesse insgesamt gemeint. Je nach sozialpolitischen Vorgaben (policies of care) sind die Konfigurationen verschieden, in denen Versorgung gestaltet und gesteuert wird und die Erbringung von sozialen und gesundheitlichen Dienstleistungen erfolgt.

In Großbritannien wird der Begriff Care Management im Nationalen Gesundheitsdienst gebraucht, um das Versorgungssystem im lokalen Gemeinwesen (community care) in seiner Organisation und Steuerung zu bezeichnen, insbesondere das Management der fallweisen Versorgung (case management). Es ist die Aufgabe der lokalen öffentlichen Sozialdienste, bei Versorgungsbedürftigkeit von behinderten oder alten Menschen den individuellen Bedarf einzuschätzen, die Abhilfen zu planen und ein Bündel notwendiger Leistungen (package of care) zu schnüren, die dann von anderer Seite erbracht werden. Zu diesem Zweck verfügt der Dienst über ein Budget und disponiert in jedem Einzelfall über die Mittelverwendung im Rahmen des insgesamt verfügbaren Budgets. Im Rahmen des Care Managements erfolgt eine Kontrolle und Evaluation der Leistungserbringung.

Vom amerikanischen System der Gesundheitsversorgung her haben sich auch in Europa Steuerungskonzepte verbreitet, die unter dem Stichwort »Managed Care « neue strukturelle und ablauforganisatorische Verbindungen von Kostenträger und Leistungserbringern, von Versicherung und Versorgung zum Inhalt haben. "Managed Care ist die Anwendung von Managementprinzipien, die zumindest partielle Integration der Leistungsfinanzierung und -erstellung sowie das selektive Kontrahieren der Leistungsfinanzierer mit ausgewählten Leistungserbringern. Ziel ist die effiziente Steuerung der Kosten und Qualität im Gesundheitswesen. « (Amelung/Schumacher 2004) Managed Care verschafft den Leistungsfinanzierern und den Konsumenten gegenüber den Leistungserbringern mehr Gewicht und Spielraum in der Gestaltung der gesundheitlichen Versorgung.

Gegenstand von Care Management sind allgemein die Strategien, die Aufbauorganisation (Strukturen), die Arrangements (care arrangements) und die Ablauforganisation (Versorgungspfade, vgl. z. B. Johnson 2002) in der humandienstlichen Versorgung. Dabei sind die informellen Weisen sozialer und gesundheitlicher (Selbst-) Versorgung zu berücksichtigen. Die Verknüpfung professioneller Aufgabenerledigung mit den Eigenleistungen von Menschen und ihrer Unterstützung durch Angehörige, Nachbarn und freiwillige Helfer wird der Forderung nach Wirtschaftlichkeit und nach Lebensnähe von Diensten gerecht. Insbesondere in der ambulanten pflegerischen Versorgung bauen fachdienstliche Arrangements auf persönliche und familiäre "care arrangements « und verbinden sich mit ihnen. Die häusliche Versorgungsarbeit, die in familiären und anderen lebensgemeinschaftlichen Arrangements zumeist von Frauen geleistet wird, war und ist fundamental für das Funktionieren der formalen Versorgungssysteme (Daly 2002, Daly/Rake 2003).

Im Betrieb sozialer und gesundheitlicher Versorgung besteht ein Zusammenhang zwischen dem fallübergreifenden Care Management und dem individuellen Case Management. Die vertikal (top-down) zu regelnde und zu steuernde Ablauforganisation und die einzelfallbezogen horizontal zu organisierenden Vernetzung im Leistungsgeschehen sind wechselseitig aufeinander abzustimmen. Das Versorgungsmanagement ermöglicht eine angemessene Fallsteuerung; das Management im Einzelfall konkretisiert und optimiert die Versorgung.

Wolf Rainer Wendt

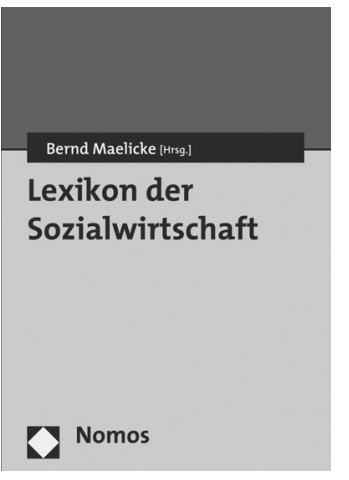

Quelle: Bernd Maelicke (Hg.): Lexikon der Sozialwirtschaft. Nomos Verlagsgesellschaft, Baden-Baden 2007. 1.128 Seiten. 98,- Euro. ISBN 978-3-8329-2511-6. Seite $172 \mathrm{f}$. 
kurzfristigen Interventionen und andauernden Erfordernissen in der eigenverantwortlichen Lebensgestaltung von Menschen überwinden müssen. Je mehr sie aktiv beteiligt sind, wenn Dienstleistungen für sie bereitgestellt und passend zugeschnitten werden, desto eher dürfte eine vorsorgende Unterstützung gelingen. Dahingehend diskutiert man international die Einbeziehung der Nutzer von personenbezogenen Dienstleistungen in deren Ausfertigung (Schehrer/Sexton 2010). Gesucht ist eine "personalisierte " Gestaltung von Versorgung.

Diese Absicht bringt eine Ermächtigung zur Mitgestaltung und eine Verantwortungsteilung in der Aufgabenerfüllung mit sich. Dem Einzelnen wird die Unsicherheit nicht abgenommen, die den Wechselfällen seines Lebens anhaftet. Eine sozialprofessionelle Begleitung folgt diesen Wechselfällen flexibel und bietet somit eine Absicherung in der individuellen Daseinsvorsorge. In ihr wird nicht selten an eine stationäre Unterbringung gedacht, weil man Unsicherheit und Risiken begegnen will. Angehörige eines Pflegebedürftigen glauben auf sie zukommenden Belastungen nicht gewachsen zu sein; Eltern nehmen für ihr Kind einen Internatsplatz in Anspruch, weil sie eine Fehlentwicklung fürchten. Den Sorgenden wird ihre Unsicherheit kaum mit einer vereinzelten Hilfestellung und Beratung abgenommen; vielmehr stützt ein ständig erreichbarer Rat - auch wenn er nur gelegentlich benötigt wird.

\section{Versorgung in zeitlicher Abstimmung}

Sprechen wir von »integrierter Versorgung «, meinen wir eine Verbindung und Vernetzung von (gesundheitlicher) Versorgung über herkömmliche Abgrenzungen hinweg. Integration ist aber auch im Verhältnis von institutioneller Fremdsorge und Eigensorge von Menschen angebracht. Dann geht es nicht um ein abgestimmtes Nebeneinander und Nacheinander von Behandlungen. Im »continuum of care « ist ein Miteinander vorausschauend zu organisieren, in dem sich versorgende Stellen und sorgende Personen aufeinander verlassen können. Die ist im Krankheitsmanagement von Chronikern nötig, in der Begleitung von Familien mit vielfältigen Belastungen oder in der Ange- hörigenpflege, für die auf längere Sicht die häusliche Lebensgestaltung eine komplementäre Abstützung braucht.

In dergleichen Problemlagen ist ein Case Management gefragt, weil mit ihm über akute Erfordernisse hinaus Hilfen kontinuierlich zusammengeführt werden. Gesteuert wird ein "continuum of care", das nicht einfach Dienste verbindet, sondern in jedem Einzelfall informell sorgendes Handeln und formelle Versorgung(smöglichkeiten) zielgerichtet abstimmt. Das akut Notwendige ist inbegriffen. In der Kontinuität der Sorge besteht die Organisationsaufgabe. (Evashwick 2005, 1)

Eine auf den persönlichen oder familiären Lebensverlauf abgestimmte Versorgung erfordert ein Zeitregime des »Wer macht was wann«. Vielen Menschen mangelt es an einer angemessenen Zeiteinteilung und Zeitverwendung. Sie müssen temporale Orientierungen erst einmal lernen oder in einer veränderten Lebenssituation neu gewinnen. Temporale Orientierungen sind Einstellungen, die in der Alltagsbewältigung gebraucht werden. Man muss planen können, kurzfristig und längerfristig disponieren und Zeitkontrolle besitzen. Temporale Orientierungen »basieren auf Lernerfahrungen in konkreten sozialen Kontexten « (Morgenroth 2008, 93).

In der alltäglichen Sorge entwickeln gerade Menschen in schwierigen Lebensverhältnissen oft ein besonderes Geschick in ihrer Zeiteinteilung. Alleinerziehende berufstätige Mütter disponieren über ihre Aufgaben im Tagesverlauf sehr genau, damit Zeit für ihre Kinder bleibt. Nicht viel anders ist es, wenn beide Eltern berufstätig sind und im Zeitdruck des Arbeitslebens "Zeit für Beziehungen « herausschlagen müssen. (Heitkötter u. a. 2009) In der Angehörigenpflege kommt es darauf an, dass in der häuslichen Versorgung die Pflegepersonen nicht zeitlich überlastet werden und Auszeiten für sie eingeplant sind. (Ringerspacher u. a. 2009)

\section{Leistungs- und Lebensgestaltung als Projekt}

In perspektivischer Betrachtung stellt sich eine ambulante und häusliche humandienstliche Begleitung als ein Unterfangen dar, das vorläufig und unabge- schlossen bleibt und sich für die Wechselfälle wie den Wandel des Lebens offen hält. Es ist ein Projekt, in Teilen und im Ganzen. Familie ist heutzutage ein Projekt, Partnerschaft bleibt eines auf Zeit und auf Dauer, Bildung und Qualifizierung sind ein lebenslanges Vorhaben, Rehabilitation und Pflege können es sein.

Der professionellen Absicht zu helfen und zu bessern stehen persönliche Erwartungen und Vorhaben in der Ungewissheit gegenüber, was aus ihnen wird. Die Adressaten des Dienstes sind in das Projekt ihrer Lebensgestaltung verwickelt und darin sollten sich auch Arrangements der Unterstützung und Versorgung komplementär einpassen lassen oder daran kompensatorisch, etwa in einem Wohnprojekt, einem Lernprojekt oder einem Projekt gemeinschaftlicher Lebensgestaltung anschließen.

\section{Literatur}

Evashwick, Connie J.: The Continuum of Long-Term Care. 3rd Edition. Thomson Delmar Learning, Clifton Park, NY 2005.

Hansbauer, Peter/Hensen, Gregor/Müller, Katja/Spiegel, Hiltrud von: Familiengruppenkonferenz. Eine Einführung. Juventa, Weinheim 2009.

Heitkötter, Martina/Jurczyk, Karin/Lange, Andreas/Meier-Graewe, Ute (Hg.): Zeit für Beziehungen? Zeit und Zeitpolitik für Familien. Barbara Budrich, Opladen 2009.

Morgenroth, Olaf: Zeit und Handeln. Psychologie der Zeitbewältigung. Kohlhammer, Stuttgart 2008.

Ringerspacher, Jürgen/Herrmann-Stojanov, Irmgard/Pfahl, Svenja/Reuyß, Stefan: Zeiten der Pflege. Eine explorative Studie über individuelles Zeitverhalten und gesellschaftliche Zeitstrukturen in der häuslichen Pflege. Lit, Münster 2009.

Schehrer, Silvia/Sexton, Stephanie: Involving users in commissioning local services. Joseph Rowntree Foundation, York 2010.

Wendt, Wolf Rainer: Sorgeberatung. Gut beraten. In: Sozialwirtschaft aktuell, Ausgabe 8/2009. April 2009. S. $1-3$. 